\title{
Experimental and numerical analysis of the influence of the nozzle-to-plate distance in a jet impingement process
}

\author{
F. V. Barbosa ${ }^{1}$, S. F. C. F. Teixeira², J. C. F. Teixeira ${ }^{3}$ \\ ${ }^{1}$ F. V. Barbosa, MEtRICs I\&D Centre, Department of Mechanical Engineering, School of Engineering, University of \\ Minho, Guimarães, Portugal* \\ ${ }^{2}$ S. F. C. F. Teixeira, ALGORITMI I\&D Centre, Department of Production and Systems, School of Engineering, University \\ of Minho, Guimarães, Portugal \\ ${ }^{3}$ J. C. F. Teixeira, MEtRICs I\&D Centre, Department of Mechanical Engineering, School of Engineering, University of \\ Minho, Guimarães, Portugal \\ E-mail: "flaviab@dem.uminho.pt
}

Received 1 Dec 2019, Revised 24 Feb 2020, Accepted 27 Feb 2020

\begin{abstract}
Jet impingement is a complex heat transfer technique which involves several process variables, such as nozzle-toplate distance, jet diameter, Reynolds number, jet temperature, among others. To understand the effect of each variable, it is important to study them separately. In industrial applications that use forced convection by air jet impingement, such as reflow soldering, the correct analysis of the flow structure and accurate definition of the variables values that affect the heat transfer over the target surface leads to an increase of the process performance decreasing the manufacturing costs. To reduce costs and time, the introduction of numerical methods has been fundamental. Using a Computational Fluid Dynamics software, the number of experiments is highly reduced, being possible to focus on the phenomena that are highly relevant for the purpose of the study. In this work, the nozzle-to-plate distance $(H / D)$ variable is analyzed. This is considered one of the most important parameters since it influences the entire structure of the jet flow as well as the heat transfer coefficient over the target surface. The results present a comparison between different $H / D$ under isothermal and non-isothermal conditions for a Reynolds number of 2,000.
\end{abstract}

Keywords: Jet Impingement; Jet Flow; Nozzle-to-plate distance; PIV; SST $k$ - $\omega$ turbulence model.

\section{Introduction}

The expansion of the electronic products market has led to an increase in the complexity of Printed Circuit Boards (PCB), which causes a more complex thermal response when a PCB passes through a reflow oven [1]. Inside this equipment occurs a process known as reflow soldering process which is achieved by forced convection using the multiple air jet impingement technology [2]. This process is currently the primary process used for the attachment of electronic components to the PCB and consists of the melting of the solder paste through hot air jets, followed by a cooling process through cold jets, allowing the connection between the board and the components. However, during the production of PCBs, it was observed that inhomogeneous thermal distribution emerges during the reflow soldering leading to soldering failures [3]. In practice, defective products require additional repairs and reworking that can cause a loss of productivity of roughly $30-50 \%$ of the total manufacturing costs [4]. To enhance the convective heat transfer, minimizing the defects that results from air jet impingement, studies have been performed in order to increase the heat transfer uniformity and to improve the coverage of the impinging surface. However, the total control of all the variables identified in jet impingement is still one of the remarkable issues of thermal design of these systems [5], such as reflow soldering.
Amongst the different process variables, nozzle-to-plate distance $(H)$ is considered one of the most important geometrical parameters in jet impingement due to its strong influence on the heat transfer performance. Several studies report the effect of $H / D$ distances over an impinging surface. An experimental work performed by Garimella \& Schroeder [6] demonstrated that a decrease in the nozzle-to-plate distance leads to an increase of the heat transfer coefficients, being this effect increased by higher Reynolds numbers. According to Angioletti et al. [7], by increasing the $H / D$, a change of the vortex size at the impinging surface is observed and the vortex breakdown occurs on the near side of $r / D=1$. Regarding the stagnation region, characterized by an overall velocity near zero [8], Reodikar et al. [9] observed that the Nusselt number distribution is more uniform for lower $H / D$ values, due to the uniform velocity profile in the potential core region of the jet. Shariatmadar et al. [10] stated that large nozzle-to-plate distances decrease the heat transfer performance due to the mixing flow between the surrounding air and the jet before the contact with the impinging surface. Considering a target surface with roughness (micro pin fins), the study performed by Brakmann et al. [11] showed that the Nusselt number decreases with increasing the nozzle-toplate distance, being more pronounced in this target surface than in flat plate. Furthermore, studies performed by Lee \& Lee [12] demonstrated that the local Nusselt number increases by up to $65 \%$ at $H / D=2$. 
Due to the advancements in numerical modelling and considering all the advantages that its implementation brings to the scientific research, a Computational Fluid Dynamic (CFD) software is used to conduct the study of the influence of the nozzle-to-plate distance on the heat transfer performance. To validate the numerical model, experiments were conducted using the Particle Image Velocimetry (PIV) technique, which allows the characterization of the jet flow through the measurement of the velocity field.

This work focuses on two cases: an isothermal and nonisothermal jet impinging a flat plate. Experiments were conducted and the data obtained for an isothermal jet are used for the validation of the numerical simulation that applies the SST $k$ - $\omega$ turbulence model to predict the single jet impingement flow. The second analysis consist of a heating process where the jet temperature $\left(120^{\circ} \mathrm{C}\right)$ is higher than the target plate temperature $\left(25^{\circ} \mathrm{C}\right)$. From data obtained by the industry, the Reynolds number applied in the reflow soldering process lies in the transition region. The research conducted in jet impingement that lies in this flow region is very scarce, since the majority of the research focus on fully turbulent jets and some approach laminar flows. In that sense, a turbulence model with a Reynolds number of 2,000 is implemented. The numerical results obtained in this case were compared with experimental data, showing that the CFD tool predicts with accuracy the jet flow.

Considering the importance of the analysis of the influence of the nozzle-to-plate distance variable on heat transfer performance of jet impingement systems, this work aims to provide answers to the industry, scientifically proved.

\section{Numerical model of a jet impingement system}

\subsection{Physical domain and boundary conditions}

The numerical model, presented in Figure 1, consists of a single jet impinging a cooper flat plate surface. The air flows through a circular nozzle at a specific velocity, which depends on the Reynolds number required in the study, and at a constant temperature of about $120^{\circ} \mathrm{C}$. Since the Mach number is below 0.3, the air jet flow is considered incompressible. After the impingement, the air flow escapes through the side walls. Considering this geometry and the flow parameters, the Reynolds number obtained in this study case is about 2,000. According to Viskanta [13], this flow lies in the transition region, since at $\operatorname{Re}<1,000$ the flow field exhibits a laminar flow behavior and at $\operatorname{Re}>3,000$ it presents a fully turbulent behavior. This low Reynolds number value represents a challenge in numerical simulations that applies turbulence models to predict impinging jets.

In that sense, uniform velocity distribution was applied at inlet while no-slip condition was implemented at both nozzle and target plate. A constant temperature of $25^{\circ} \mathrm{C}$ was specified to the target surface and insulated wall was defined at the nozzle plate. Pressure outlet boundary condition with zero initial gauge pressure was applied to the open sides of the domain.

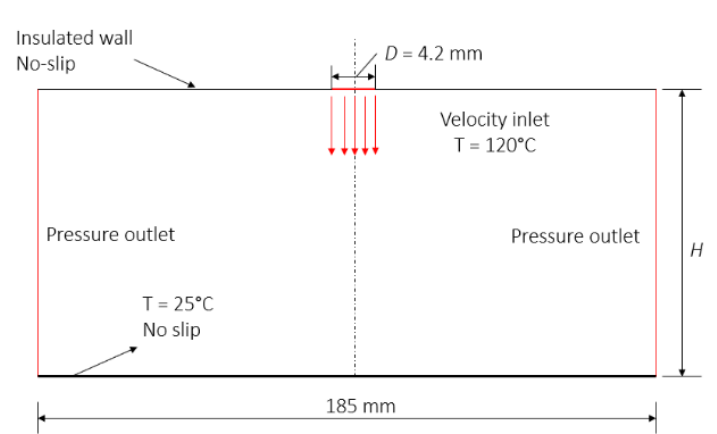

Figure 1. Physical domain of the numerical model.

\subsection{Governing Equations}

The jet impingement system will be studied in two dimensions. In that sense, the equations that define this problem are the following:

$$
\begin{gathered}
\frac{\partial u}{\partial x}+\frac{\partial v}{\partial y}=0 \\
\frac{\partial u}{\partial t}+u \frac{\partial u}{\partial x}+v \frac{\partial u}{\partial y}=-\frac{\partial p}{\partial x}+\mu \frac{\partial^{2} u}{\partial x^{2}}+\mu \frac{\partial^{2} u}{\partial y^{2}} \\
\frac{\partial v}{\partial t}+u \frac{\partial v}{\partial x}+v \frac{\partial v}{\partial y}=-\frac{\partial p}{\partial y}+\mu \frac{\partial^{2} v}{\partial x^{2}}+\mu \frac{\partial^{2} v}{\partial y} \\
\frac{\partial T}{\partial t}+u \frac{\partial T}{\partial x}+v \frac{\partial T}{\partial y}=\alpha \frac{\partial^{2} T}{\partial x^{2}}+\alpha \frac{\partial^{2} T}{\partial y^{2}}
\end{gathered}
$$

These governing equations are already simplified for an incompressible flow. "Eq. (1)" represents the continuity equation, "Eq. (2)" and "Eq. (3)" are the Navier-Stokes equations in $2 \mathrm{D}, x$-momentum and $y$-momentum respectively, while "Eq. (4)" is the energy equation. The effect of gravity, angular velocity vector and source term are neglected, since the process in study is mainly influenced by convection.

Considering the equations presented above, it is possible to identify the unknown and known variables. The unknowns are the velocity in $x$-direction, $u$, the velocity in $y$-direction, $v$, the pressure, $p$, and the temperature, $T$. The known variables are the dynamic viscosity and thermal diffusivity of the fluid, $\mu$ and $\alpha$ respectively.

\subsection{Mathematical model and numerical method}

According to several authors, it appears that the Shear Stress Transport (SST) $k$ - $\omega$ turbulence model proved to be both accurate and computing time saving in engineering applications. $\mathrm{Zu}$ et al. [14] compared different turbulence models with experimental data and mentioned that the SST $k-\omega$ model presents a better compromise between computational costs and accuracy. Later, Ortega \& Ortiz [15] verified a good agreement between numerical and experimental data using this turbulence model, being registered a maximum deviation of $8 \%$. In addition, Wen et al. [16] confirmed the good accuracy of the SST $k$ - $\omega$ model especially in the stagnation zones of the jets. A recent study performed by Penumadu \& Rao [17] revealed that the heat transfer characteristics are well predicted by SST $k-\omega$ model, mainly due to its ability to handle accurately regions with high pressure gradients.

Due to the advantages pointed out by several studies mentioned above, the SST $k-\omega$ model was selected for the numerical simulation of the jet impingement. This model, developed by Menter [18], applies the $k$ - $\omega$ model in the near wall region and switches to the $k-\varepsilon$ model in the far field, combining the advantages of both models. $k$ - $\omega$ model performs much better 
than $k-\varepsilon$ model for boundary layer flows, however, it is excessively sensitive to the freestream value of $\omega$ which is not the case of the $k-\varepsilon$ model. The combination between the SST and the $k-\omega$ models improves the near wall treatment since it gradually switches from a classical low-Reynolds formulation on fine meshes to a log-wall function formulation on coarser grids [19]. To describe the flow near a wall, the SST $k-\omega$ model uses a lowReynolds number approach allowing the consideration of the details in the viscous sublayer [20]. The equations of the turbulence kinetic energy, $k$, and the specific dissipation rate, $\omega$, are presented in "Eq. (5)" and "Eq. (6)", respectively:

$$
\begin{aligned}
& \frac{\partial}{\partial t}(\rho k)+\frac{\partial}{\partial x_{i}}\left(\rho k u_{i}\right)=\frac{\partial}{\partial x_{j}}\left(\Gamma_{k} \frac{\partial k}{\partial x_{j}}\right)+G_{k}-Y_{k}+S_{k} \\
& \frac{\partial}{\partial t}(\rho \omega)+\frac{\partial}{\partial x_{i}}\left(\rho \omega u_{i}\right)=\frac{\partial}{\partial x_{j}}\left(\Gamma_{\omega} \frac{\partial \omega}{\partial x_{j}}\right)+G_{\omega}-Y_{\omega}+S_{\omega}
\end{aligned}
$$

where $\Gamma$ represents the effective diffusivity, $G$ the generation and $Y$ the dissipation of the corresponding variables. $D_{\omega}$ is the cross-diffusion term while $S_{k}$ and $S_{\omega}$ are the user-defined source terms [16].

To solve the pressure-velocity coupling, the SIMPLE algorithm was applied. This algorithm uses a relationship between velocity and pressure corrections to enforce mass conservation and to obtain the pressure field [21]. Regarding the spatial discretization of momentum, a second-order upwind was imposed while for the dissipation rate and turbulent kinetic energy, a first order upwind was applied. The computational models were solved using a transient formulation based on a first-order implicit method and the convergence criterion of $1 \mathrm{E}-3$ for continuity, momentum and turbulence equations and 1E6 for energy equation.

\subsection{Mesh sensitivity}

The mesh sensitivity analysis is extremely important to determine the accuracy of the predictions as a function of the mesh quality. To perform this analysis two parameters were considered, the number of elements and the bias factor. The bias factor is the ratio of the largest to the smallest element, i.e. a higher bias factor implies a greater refinement close to the walls. In that sense, a bias factor of 4 and 8 was implemented in order to refine the mesh near the target surface. However, a compromise between the mesh refinement close to the walls and the quality of the mesh must be ensured. Skewness, aspect ratio and element quality factors were used as criteria for element's evaluation: Skewness determines how close to the ideal a face or cell is (i.e. equilateral or equiangular), a value close to zero defines equilaterality of the element; aspect ratio is the ratio of the longest edge length to the shortest edge length, being one for an equilateral cell; element quality represents the ratio of the volume to the sum of the square of the edge lengths for $2 \mathrm{D}$ elements or square roots of the cub of the sum of the square of the edge lengths for $3 \mathrm{D}$ elements, in which a value of 1 defines a perfect cube or square [22]. Nevertheless, ensuring the recommended values is difficult since a refinement implies smaller elements close to the walls and higher elements in the remaining domain.

To analyze the meshes, the quality parameters of each one, the computation time, as well as the $y+$ value, i.e. the dimensionless distance of the first node to the wall [16], are presented in Table 1. Looking at the computation time, it is observed that the higher the number of elements, the greater the simulation time as expected. In addition, it appears that increasing the bias factor do not increases the complexity of the mesh. In contrary, it seems to improve the convergence of the results, thus the simulation time is $6 \mathrm{~h}$ higher for a mesh with a lower bias factor. Regarding the mean wall $y+$, it seems that its value is given by a combination between the number of elements and the refinement close to the wall.

To determine the optimum grid size, the Nusselt number variation over the target surface was analyzed and compared. Since a larger Nusselt number represents a more effective convection [23], this property allows to analyze the heat transfer performance of the jet impingement. This property represents the ratio between convection and conduction across a fluid (Eq. 7).

$$
\mathrm{Nu}=\frac{h \cdot D}{k_{\text {air }}}
$$

where $h$ is the heat transfer coefficient, $D$ the jet diameter and $k_{\text {air }}$ the thermal conductivity of the air. The heat transfer coefficient is obtained by "Eq. (8)" which represents the ratio between the convective heat flux $(\dot{q})$ and the temperature difference between the target surface $\left(T_{\text {wall }}\right)$ and the air jet at the inlet $\left(T_{j e t}\right)$ [26-28].

$$
h=\frac{\dot{q}}{T_{\text {wall }}-T_{j e t}}
$$

Table 1. Mesh Properties.

\begin{tabular}{lccccc}
\hline Mesh & $\begin{array}{c}1 \\
(\text { coars } \\
e)\end{array}$ & $\begin{array}{c}2 \\
(\text { mediu } \\
m)\end{array}$ & $\begin{array}{c}3 \\
\text { (fine) }\end{array}$ & $\begin{array}{c}4 \\
\text { (fine) })\end{array}$ & $\begin{array}{c}5 \\
\text { (fine) }\end{array}$ \\
\hline $\begin{array}{l}\mathrm{N}^{\mathrm{o}} \text { of } \\
\text { Element } \\
\mathrm{s}\end{array}$ & $\begin{array}{c}10,0 \\
80\end{array}$ & 36,800 & $\begin{array}{c}124,50 \\
0\end{array}$ & $\begin{array}{c}124,50 \\
0\end{array}$ & $\begin{array}{c}336,00 \\
0\end{array}$ \\
$\begin{array}{l}\text { Bias } \\
\text { factor }\end{array}$ & 8 & 8 & 8 & 4 & 4 \\
$\begin{array}{l}\text { Skewnes } \\
\mathrm{s}\end{array}$ & & & $1.30 \times 10^{-10}$ & & \\
$\begin{array}{l}\text { Aspect } \\
\text { Ratio }\end{array}$ & 1.93 & 1.93 & 1.93 & 1.56 & 1.88 \\
$\begin{array}{l}\text { Element } \\
\text { Quality }\end{array}$ & 0.82 & 0.82 & 0.82 & 0.90 & 0.83 \\
$\begin{array}{l}\text { Mean } \\
\text { wall y }\end{array}$ & 5.16 & 2.66 & 1.31 & 2.2 & 1.03 \\
$\begin{array}{l}\text { Simulati } \\
\text { on Time } \\
(t=1 \mathrm{~s})\end{array}$ & 30 & $5 \mathrm{~h}$ & $11 \mathrm{~h}$ & $17 \mathrm{~h}$ & 3 days \\
\hline
\end{tabular}

As it can be observed in Figure 2, the Nusselt number profile over the target surface is different in the five cases. These results prove that the SST $k$ - $\omega$ turbulence model is sensitive to the quality of the mesh. Increasing the number of elements, the stagnation point is clearly identified, in contrast to the coarse and medium grids that are not able to predict this point. Regarding the variation of the Nusselt number over the plate, it is observed that a coarse grid presents lower Nusselt number values close to the jet axis, while the medium one has difficulties to predict the stagnation point, showing higher Nusselt number values in the vicinity of this point. Moreover, it is observed that the mesh with 124,500 (bias = 8) presents similar values compared with a mesh with 336,000 elements and a bias factor of 4 . The results demonstrate that, to ensure a good 
accuracy of the simulation with less elements, a bias factor must be applied. These observations are extremely important since the higher the number of elements, the greater the computation time and the memory required. In addition, the data demonstrate that the improvement of the results using a mesh with more elements does not justify the simulation time required. Focusing on the Nusselt number obtained by the fine grid at the stagnation point, it is observed that the maximum value lies between the range $20<\mathrm{Nu}<30$. These values are in accordance with numerical and experimental works found in literature that apply a $\operatorname{Re}=2,000$ or close to this value [27][28]. Considering the analysis presented above, it is suggested that Mesh 3 presents the best conditions to conduct the numerical simulations.

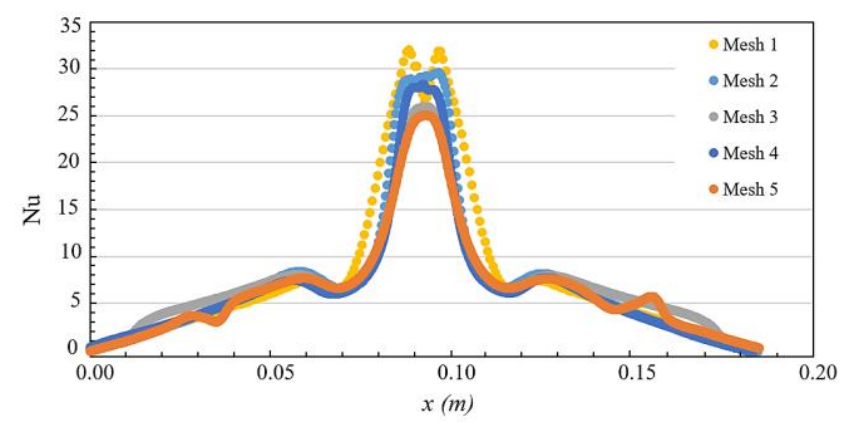

Figure 2. Nusselt number over the surface for different meshes.

\section{Experimental Apparatus}

To perform a jet flow analysis, an experimental research will be performed on a purpose-built test facility which has been commissioned, using a Particle Image Velocimetry (PIV) system. This method, depicted in Figure 3, allows the measurement of the velocity field of the jets using a doublepulse Nd:YAG laser and a CCD camera. The laser generates a two-dimensional laser sheet which illuminates the measurement region from the exit of the nozzles to the target plate. The flow seeding is ensured by olive oil tracer particles with a diameter between 1-3 $\mu \mathrm{m}$ introduced inside the system using a smoke generator. This diameter complies with the requirement of an accurate air flow tracking presented by Melling [29]. The seeding particles follow the instantaneous motion of the air and scatter the light that will be captured by the CCD camera with a pixel resolution of $2560 \times 2160$ (5.5 Megapixel). The CCD camera captures two consecutive images spaced by a short time interval in order to visualize the displacement of the seeding particles from one image to the next. This time between pulses was defined through an analysis of the velocity field accuracy using different times values. The data acquisition and processing of the images are performed by the software Dynamic Studio which divides the images into interrogation areas and applies mathematical correlation to obtain velocity vectors [30].

The experimental apparatus, whose scheme is presented in Figure 4, consists of a ventilator (1) connected to a diffuser (2) that directs the air to a stabilization chamber (3) in order to reduce the turbulence. A flow regulator allows the control of the air velocity that will flows through the orifice nozzle (5). An acrylic pipe is attached to the stabilization chamber, inside which a honeycomb structure (4) was placed to ensure the uniformization of the flow. The PIV laser and camera were positioned perpendicularly at a distance that allows to capture the flow from the exit of the nozzles to the target surface. The probe of the smoke generator is introduced inside the stabilization chamber to ensure a correct uniformization of the seeded flow throughout the pipe.

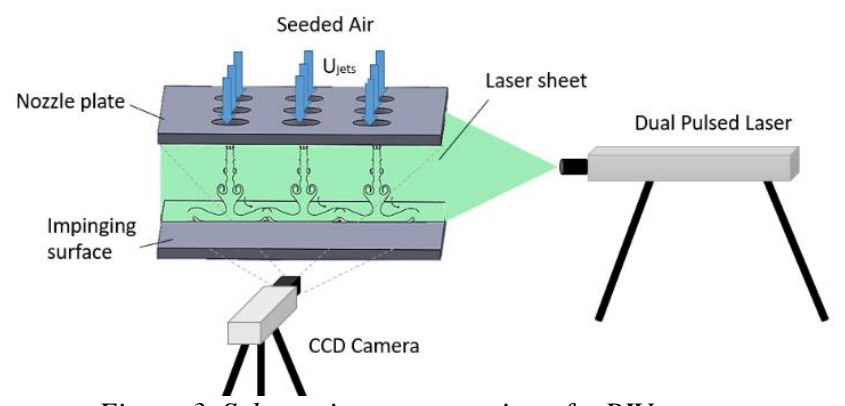

Figure 3. Schematic representation of a PIV system.

The target plate was placed above the nozzle plate on a table that allows to control the spacing between the plate and the nozzle. These experiments were conducted under isothermal conditions (ambient temperature of about $20^{\circ} \mathrm{C}$ ) and the nozzle-to-plate was varied from $2 D$ to $7 D$. To ensure a correct analysis of the PIV results 300 images were analyzed for each case and the time-averaged velocity profile was obtained. Since velocities around $7 \mathrm{~m} / \mathrm{s}$ are expected, a time between pulses around $100 \mu \mathrm{s}$ was defined as a fixed parameter of the laser. However, it was verified that decreasing the nozzle-to-plate distance, higher turbulence occurs between the target and nozzle plate, to minimize measurements noise, the time between pulses was decreased from $100 \mu$ s to $80 \mu$ s for $2 \leq H / D \leq 4$.

The results obtained will be compared with the numerical results performed with ANSYS FLUENT software. With this analysis it is expected to validate the numerical results and to prove that the SST $k-\omega$ model is suitable for the prediction of a single jet impingement in a transition regime. After this validation, the comparison between the flow profile of an isothermal and non-isothermal jet will be performed taking into consideration the influence of the nozzle-to-plate distance. A Reynolds number of 2,000 at the exit of the nozzle was ensured throughout the experiments. The control of the air flow rate was performed using the fan flow regulator.

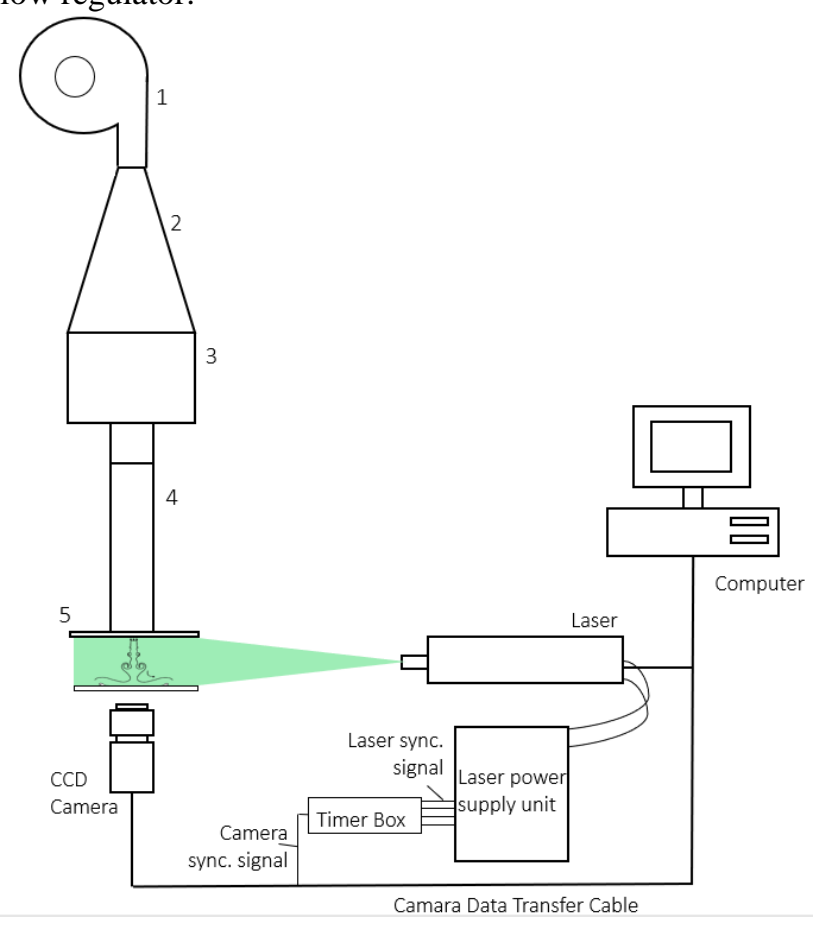

Figure 4. Experimental Apparatus. 
The resultant random error related to the PIV measurements was obtained through a statistical analysis of the data for 300 images, each case. This value was obtained from the square root of the squared random error of velocity components in $x$ and $y$ directions. Considering that these error components are independent and follow a normal distribution, for a confidence level of $95 \%$ [31], the maximum random error obtained for the maximum velocity recorded over the target surface was approximately $8 \%$. The uncertainty of the measurement is expected to decrease with the increase of the sample size. According to [32], 2000 samples seems to be a good number to reduce the uncertainty of the time-averaged velocities, $u$ and $v$.

\section{Results and Discussion}

\subsection{Flow Characterization}

The flow characterization of an isothermal jet is described in this section. The results obtained experimentally and numerically were analyzed and compared for different $H / D$.

Figure 5 shows the variation of the velocity profile of an isothermal jet at different nozzle-to-plate distances. The experimental results (right-hand side of each velocity profile) are compared with those obtained numerically (lefthand side). Even if smooth differences can be observed, generally, both approaches present a good agreement.

The different regions of an impinging jet, according to Martin [33], are identified and presented with detail in Figure 6: the initial free jet, the decaying jet, the stagnation region and the wall jet. The first region is generated at the nozzle exit where the maximum velocity is measured and characterized by the interaction between the jet and the surrounding air, inducing entrainment of mass, momentum and energy [13]. The potential core, defined by Livingood \& Hrycak [34] as the distance from the nozzle exit to the position where the jet velocity decays $95 \%$ of its maximum velocity, is also clearly identified both numerically and experimentally. From Figure 5, it seems that increasing $H / D$ leads to a decrease of the potential core length due to the higher dissipation of the jet velocity, showing that low $H / D$ generated uniform velocity profile in this region. The end of the core region is followed by the beginning of the decaying region which is characterized by the linear variation of the axial velocity and the jet width with the axial position [35]. As the flow gets closer to the wall, it loses axial velocity and turns, generating a stagnation region in which the overall velocity is near zero [8]. This point was clearly identified experimentally at $H / D$ equal to 2,6 and 7 , however, in the other cases, the larger velocity vectors seems to cover-up this point. Numerical data show a region close to the jet axis were the velocity is near zero. The last region, the wall jet, is identified once the air jet impacts on the target surface. After the contact with the plate, the flow is divided into two streams moving in opposite radial directions along the surface, being observed a change of the flow direction from axial (vertical axis $y$ ) to radial (longitudinal axis $x$ ) direction [36]. The wall jet region is characterized by a maximum radial velocity which moves away from the plate, since the flow in this region is mainly radial with a growing boundary layer [8]. Increasing the distance from the jet axis, the wall jet entrains flow and increases in thickness, while the flow velocity decreases. In the vicinity of the stagnation point, an increase of the velocity is detected in all cases which can be explained by a rapid acceleration of the flow due to larger pressure gradients. Large heat transfer coefficients can be obtained in this specific region, in the transition from the laminar to turbulent boundary layer [37]. The separation of the flow occurs where the boundary layer leaves the surface of the plate and seems to occur closer to the impinging point at low nozzle-to-plate distances.

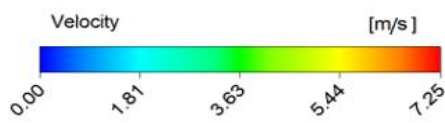

$H / D=2$

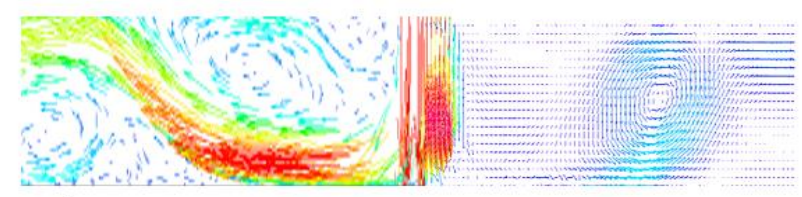

$H / D=3$

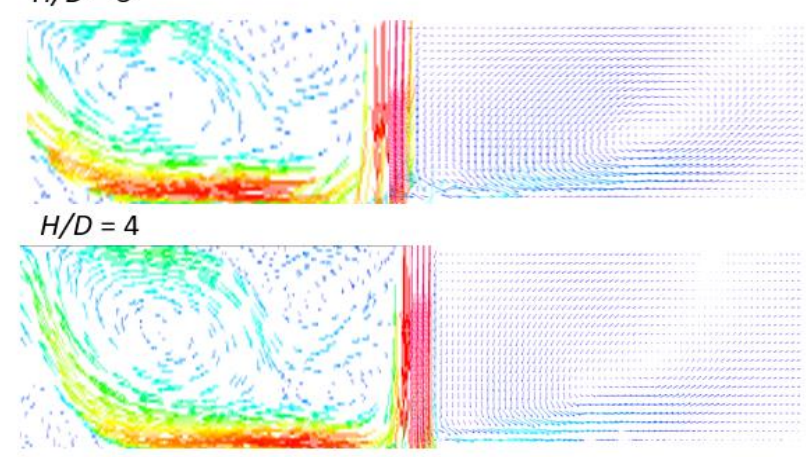

$H / D=5$

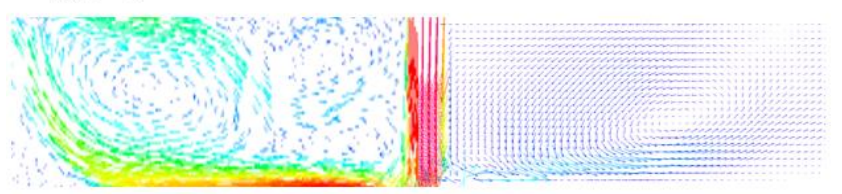

$H / D=6$

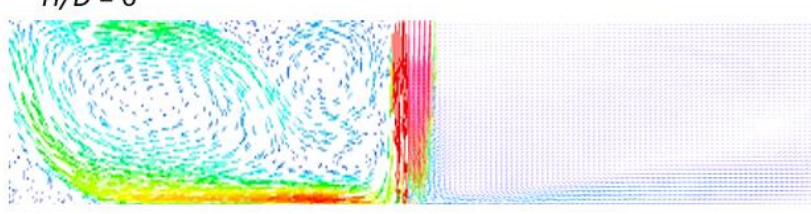

$H / D=7$

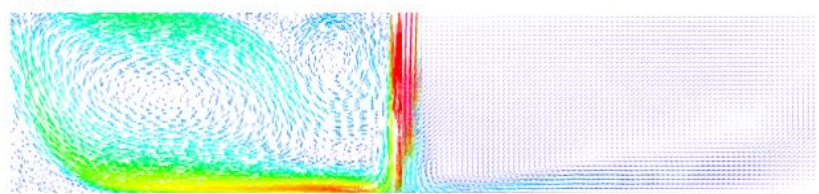

Figure 5. Jet flow velocity profile. The right-hand side presents the experimental data while the left-hand side depicts the results obtained numerically.

As Figure 5 shows, the wall jet thickness increases with decreasing the nozzle to plate distance due to higher local pressure induced by strong interactions between the jet and the surrounding air. Furthermore, results demonstrated that the wall jet region increases in the radial direction with the increase of $H / D$, which is in accordance with other confined jet impingement studies at higher Reynolds numbers [7] [37]. Focusing on the vortices generated on both sides of the jet axis, it seems that the higher the nozzle-to-plate distance, the lower the magnitude of the vortices generated, which is essentially due to the larger space for the flow to develop. 


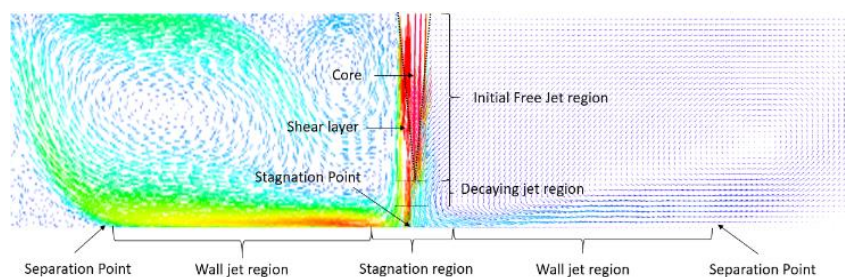

Figure 6. Flow regions of an impinging jet.

Through the previous analysis, it seems that both numerical and experimental results predict with accuracy the flow structure of a single jet in the transition region. Focusing on quantitative data, the time-averaged velocity over the target surface at different $H / D$ values was plotted and presented in Figure 7.

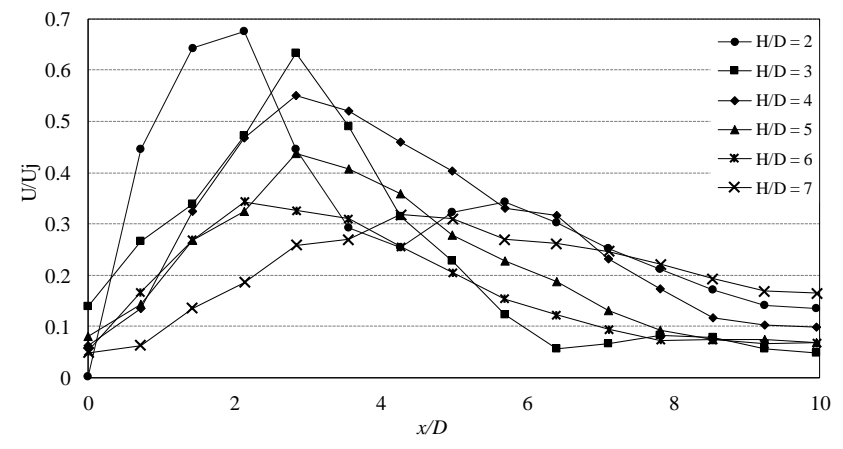

Figure 7. Time-averaged velocity over the target surface at different $H / D$ and $R e=2,000$.

Considering an air jet at ambient temperature $\left(\approx 20^{\circ} \mathrm{C}\right)$ impinging a flat plate at the same temperature, the results obtained experimentally (Figure 7) shows the evolution of the nondimensional velocity, $U / U j$, where $U$ is the timeaveraged velocity obtained by PIV measurements and $U j$ the maximum velocity recorded at the nozzle exit, over the target surface. The maximum velocity is achieved for a nozzle-toplate value of 2 , at a distance from the jet axis of approximately $x / D=2$. This is in accordance with the jet flow structure presented in Figure 5, small confined spaces induce a stronger interaction between the surrounding air and the vortices generated by the jet impingement, leading to higher velocities over the wall. Results also show that the higher the nozzle-to-plate distance, the lower the velocity in the vicinity of the stagnation point. The maximum value is recorded in all cases at a distance from the jet axis $(x / D)$ between 2 and 4, increasing with the increase of $H / D$. Moreover, as it was expected, the jet wall increases with increasing $H / D$, leading to a smoother decrease of the velocity throughout the target plate. For $H / D=2$, an interesting phenomenon is observed. After reaching the maximum velocity, its value starts to decrease, achieving a minimum velocity at approximately $x / D=4$. From this point, as opposed to the other cases, the velocity rose again achieving a second maximum point at about $x / D=6$. This secondary point was identified in several studies, mentioned in Zuckerman \& Lior [35] and Viskanta [13], being attributed to the transition of the boundary layer from laminar to turbulent flow along the wall. A secondary velocity peak was also identified in $H / D=3$ at approximately $x / D=8$. However, with the increase of the nozzle-to-plate distance, the interactions between the jet and the surrounding air in the confined space decreases, and since the Reynolds number is low, no secondary peak was identified at higher $H / D$ values. Viskanta [13] summarized the influence of the nozzle-to-plate distances smaller than the jet potential core length on the radial distribution of convective heat transfer coefficient and pointed out three main factors: (a) the laminar boundary layer behavior under strongly accelerated surrounding flow in the vicinity of the stagnation point; (b) the interaction of large-scale turbulence induced in the mixing zone; but also (c) the transition of the boundary layer from laminar to turbulent over the wall jet.

Regarding the stagnation point, it is detected with a higher accuracy at $H / D=2$, with a velocity value very close to zero at the jet axis, as it is expected. However, this is not observed in the other cases. This can be explained by the fact that, to detect this point with accuracy, an interrogation area with a higher resolution than the one used in this experiment $(32 \times 32$ pixels $)$ must be used. To reduce the lag between the expected value $(U=0)$ and the ones obtained experimentally, the focus angle of the camera should be reduced to the zone that we intend to analyze, in this case the stagnation region. However, since in this experiment it is expected to characterize all the flow, from the nozzle exit to the target plate, the focus angle must be large. Another reason to explain this lag is related to factors that can generate systematic errors such as the concentration of particles as well as the time between pulses. These are two fundamental factors that can decrease the accuracy of the PIV measurements. It was verified that even if the same Reynolds number was maintained throughout the experiments, since higher velocities were recorded over the wall region for lower nozzle-to-plate distance, we had to decrease the time between pulses to increase the accuracy of the results. Meaning that, for $2 \leq H / D \leq 5$ a lower time between pulses was implemented compared with $H / D$ of 6 and 7 . This adjustment of the time between pulse in function of the flow velocity is crucial to ensure accurate results.

\subsection{Numerical model validation}

The experimental data was used for the validation of the numerical model. In that sense the time-averaged velocity over the target surface at different nozzle-to-plate spacings for a Reynolds number of 2,000 obtained numerically were compared with the experimental results. Observing in detail Figure 5 which shows the velocity field of a single jet flow $(\mathrm{Re}=2,000)$ at a different nozzle-to-plate distance, it is clear that the SST $k$ - $\omega$ model do not predict with accuracy the potential core in the case of $H / D$ equal to 6 and 7 . While the experimental data demonstrated clearly a decrease of the potential core length with the increase of the nozzle-to-plate distance, this decrease is smoother in the case of the numerical data. As mentioned previously, the increase of $H / D$ leads to a decrease of the potential core length due to the higher dissipation of the jet velocity. However, this dissipation of velocity for higher $H / D$ values is not accurately predicted numerically. This shows that the numerical model must be improved to increase the accuracy of the prediction in the stagnation region. Furthermore, regarding the wall jet region, the decrease of the wall thickness with increasing $H / D$ values is easily observed through the numerical results.

From the flow velocity over the target surface, the results obtained numerically were plotted and presented in Figure 8. The secondary velocity peak was identified in the case of $H / D=2$ between $6 \leq x / D \leq 8$, even if this value is lower than the one obtained experimentally $U / U_{j}=0.2$ against $U / U_{j}=$ 0.34. Regarding the stagnation point, it was not predicted with accuracy in any case, a value of $U / U_{j}=0.1$ was obtained 
instead of 0 . However, this point was also difficult to identify experimentally.

Looking at the maximum velocity value, it was recorded in the vicinity of the stagnation point, as it was expected, however, in contrast to the experimental data, this peak was detected at $x / D=1.2$ for all the nozzle to plate distances. Regarding the maximum velocity, its value seems to decrease with increasing $H / D$, as expected, however this decrease is more pronounced in the experimental results. For $H / D=2$, the nondimensional velocity $U / U_{j}$ obtained both experimentally and numerically is close to 0.7 , while in the other cases, it is predicted to be around 0.6 by the turbulence model. In contrast, the experimental results show a decrease from 0.7 at $H / D=2$ to approximately 0.3 at $H / D=7$. This discrepancy between numerical and experimental results show that the effects of the surrounding air on the jet flow development are more pronounced experimentally. Even if the measurement zone is enclosed by an acrylic box, it is difficult to ensure constant temperature and humidity conditions throughout the experiments.

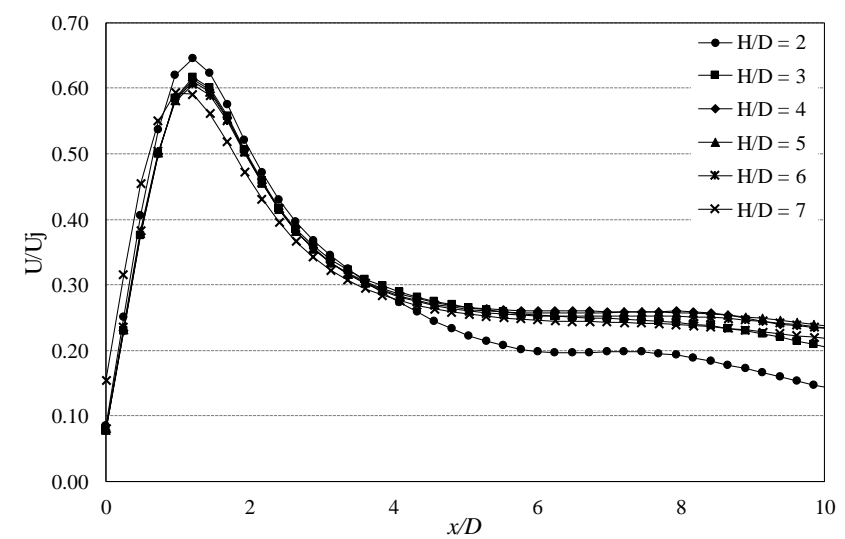

Figure 8. SST $k$ - $\omega$ velocity over the target surface at different $H / D$ and $\operatorname{Re}=2,000$.

The variation of $U / U_{j}$ throughout the jet axis in the case of $H / D$ equal to 2 and 7 was presented in Figure 9. The profile predicted numerically is very close to that obtained experimentally, with a maximum and constant velocity recorded close to the jet axis (maximum $y$ value). After leaving the nozzle, the air jet started to entrain surrounding air, decreasing the velocity which became steeper near the target plate. This velocity decrease was more pronounced at $H / D=7$, due to the lower potential core length compared with $H / D=2$. The minimum velocity value was achieved at the stagnation point, as expected. However, it is clear that the velocity profile obtained numerically is more uniform compared with the experimental ones. This deviation is essentially due to external factors, discussed in section 4.1, which are not considered by the numerical simulation. Moreover, it is verified that higher velocities are recorded over the jet axis at lower nozzle-to-plate spacing, which is in agreement with the previous results. A lower $H / D$ leads to an increase of turbulence between the main jet and the wall jet.

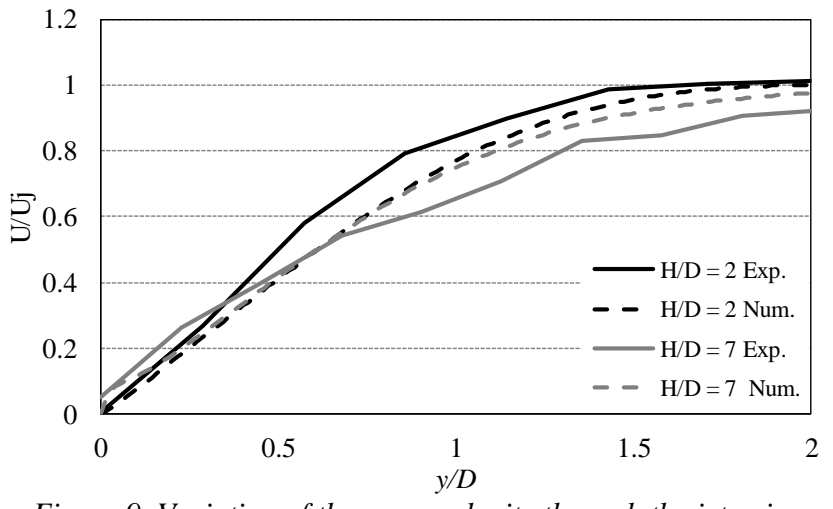

Figure 9. Variation of the mean velocity through the jet axis.

The comparison between numerical end experimental results show a good agreement between the two approaches, essentially regarding qualitative data. The SST $k$ - $\omega$ model was able to predict with accuracy the jet flow structure; however, it fails in the prediction of the potential core. The maximum velocity was predicted in the vicinity of the stagnation point and decreases with increasing $H / D$. Focusing on the quantitative data, the maximum velocity was accurately predicted in the case of $H / D=2$ while for higher $H / D$ values it was over predicted. The secondary peak was also identified numerically even if the value was under predicted compared with the experimental data. In general, we can conclude that the SST $k$ - $\omega$ model presents good qualitative predictions of the single jet impingement flow at Reynolds number in the transition region. If quantitative results are important for the analysis, this turbulence model is more accurate for higher Reynolds numbers.

\subsection{Non-isothermal jet}

After the validation of the numerical model of a single jet impingement using the SST $k-\omega$ model, a non-isothermal jet, with the same geometrical variables implemented in the isothermal jet, was analyzed numerically.

The mean velocity over the target plate obtained for a non-isothermal jet was compared with an isothermal one for the case of $H / D$ equal to 2 and 7 . The results are depicted in Figure 10.

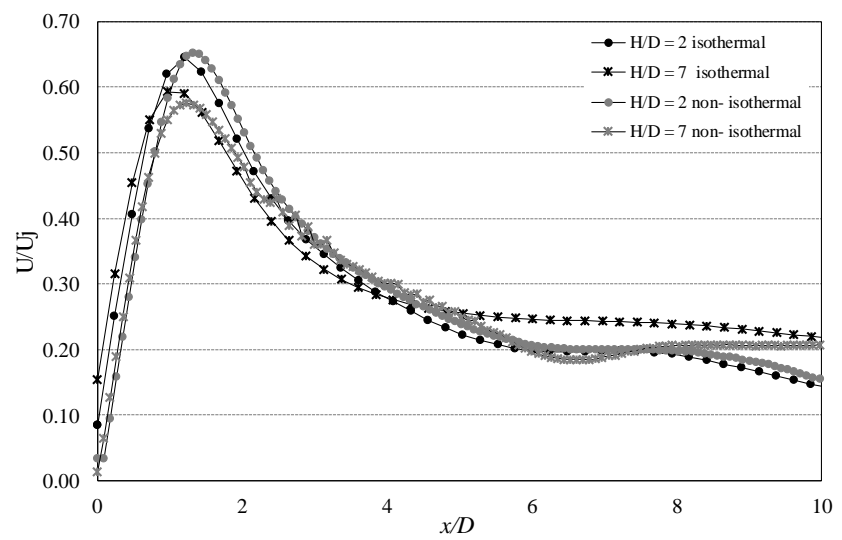

Figure 10. Comparison between the variation of the mean velocity over the target surface for an isothermal and non-isothermal jet. 
Comparing the velocity profiles for the case of an isothermal and non-isothermal jet, it is interesting to observe that the peak velocity is reached at the vicinity of the stagnation point, as expected, but at a higher distance from this point compared with the isothermal jet, $x / D \approx 1.4$, against $x / D \approx 1.2$. These results show that increasing the temperature difference between the jet and the target plate, the development of the boundary layer throughout the target plate is slightly different compared with isothermal jets. It seems that the acceleration of the radial flow in the vicinity of the stagnation point is higher in the isothermal case. However, no relevant difference is observed in nondimensional velocity $U / U_{j}$ value, being approximately 0.65 at $H / D=2$ and close to 0.6 for $H / D=7$. Focusing on the secondary peak, it was predicted at a nozzle-to-plate distance of 7 for the case of a non-isothermal jet, showing that the jet temperature and velocity leads to a higher mixing between the jet and the surrounding air.

Figure 11 and Figure 12 present the heat transfer performance of a single air jet impinging a flat plate at different nozzle-to-plate distances $(H=2 D, 3 D, 4 D, 5 D, 6 D$ and $7 D$ ), considering a Reynolds number of 2,000 (transition region). To compare the results obtained in each case, numerical data was plotted in order to analyze the variation of the Nusselt number throughout the target plate. As it is expected, the maximum Nusselt number value is registered at the stagnation point $(x / D=0)$ in all cases. The stagnation point is localized in the stagnation zone $(0 \leq x / D \leq 1)$ characterized by a very thin thermal boundary layer, leading to a large heat transfer coefficient in this region [38].

Regarding the analysis of the maximum Nusselt number, it seems that the difference between the values registered in all cases is very small. According to the research conducted by Hoffman et al. [36], the higher the Reynolds number the greater the difference between the Nusselt number values registered at the stagnation point. In their study, a higher degradation of the heat transfer is observed for $H=8.5 \mathrm{D}$ at $\operatorname{Re}=14,000$. Since the Reynolds number applied in this study is low, and considering the observations presented by [36], it seems that the effect of the nozzle-to-plate distance (H/D between 2 and 7) on the maximum Nusselt number value at the stagnation point is very smooth. These results are in accordance with Gardon et Akfirat [39] who mentioned that for impingement within the length of the potential core $(0.5<H / B<5)$ and for low Reynolds numbers $(\operatorname{Re}<2,000)$, the maximum Nusselt number registered at the stagnation point depends on the Reynolds number only. The increase of the Reynolds number leads to an increase of the vortices generated over the target surface inducing larger turbulence intensity that increase the heat transfer rate.

Increasing the distance from the stagnation region, the flow develops over the target surface, passing through a transition region where the boundary layer changes from laminar to turbulent. In this region, a secondary Nusselt number peak value was identified. Results show that it is slightly higher for lower $H / D$ and less pronounced at higher $H / D$. This behavior is also observed when fully turbulent flows are applied [40]. Gardon \& Akfirat [41] who work with laminar air jets observed that low Reynolds numbers and high nozzle-to-plate spacings lead to a lower magnitude of the secondary Nusselt number peaks. The decrease of the secondary peaks with the increase of $H / D$ can be explained by the decrease of the potential core width, since the maximum heat transfer is obtained at a nozzle-to-plate distance equal to the length of the potential core [34]. The transition region is followed by the wall jet region were a similar decrease of the Nusselt number is observed in all cases. This decrease is essentially due to lower flow velocities and high exchange of momentum between the wall jet and the surrounding air [42].

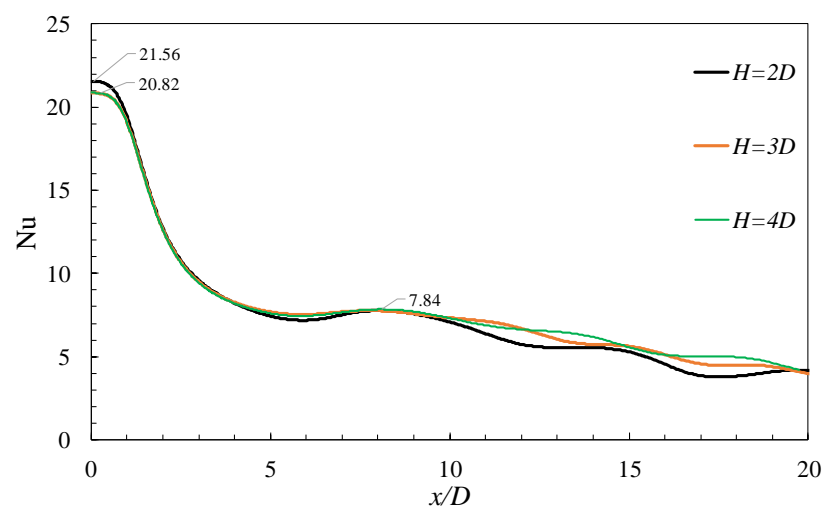

Figure 11. Nusselt number over the target surface for different nozzle-to-plate distances at $R e=2,000$ and $H / D=2,3$ and 4 .

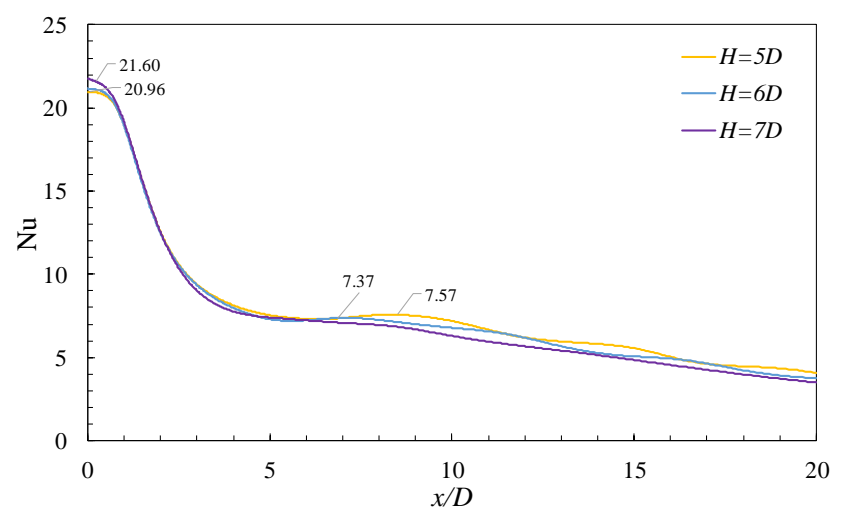

Figure 12. Nusselt number over the target surface for different nozzle-to-plate distances at $R e=2,000 \mathrm{H} / \mathrm{D}=5$, $6,7$.

To confirm that the effect of the nozzle-to-plate distance decreases with the decrease of the Reynolds number, a numerical simulation was performed under the same conditions for $\operatorname{Re}=1,000$. The variation of the Nusselt number over the impinging surface was plotted for $H / D=2$, 4 and 6 and presented in Figure 13. As it can be observed, the maximum Nusselt number value decreases with the decrease of the Reynolds number and the differences between each case is even smoother compared with the results obtained at a Reynolds number of 2,000. However, the secondary Nusselt number peak is clearly identified at $H / D=2$ but almost inexistent at $H / D=4$ and 6 . These results are in accordance with the research conducted by Gardon et Akfirat [39] and show that the heat transfer performance is increased by higher Reynolds numbers and lower $H / D$ values. 


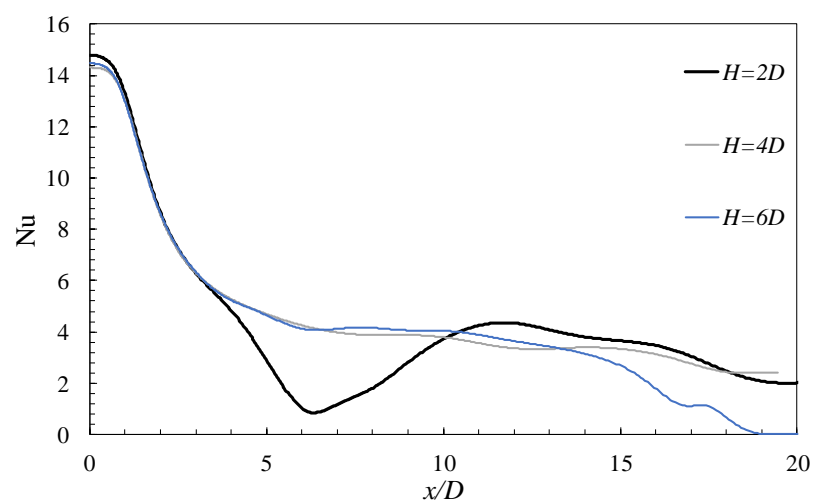

Figure 13. Nusselt number over the target surface for different $H / D$ distances at $R e=1000$.

\section{Conclusions}

This work demonstrates the influence of the nozzle-toplate distance in the heat transfer performance of a single air jet impinging a flat plate. Experiments were conducted using a PIV technique and the results were compared with numerical data to validate the model. Due to its high accuracy in predicting the jet impingement behavior, the SST $k-\omega$ turbulence model was implemented. Since this model is sensitive to the mesh quality, a mesh sensitivity analysis was performed. The results show that the accurate prediction of the stagnation point varies with the quality of the mesh as well as the maximum Nusselt number value. These observations are extremely important and demonstrate the importance of the implementation of a good mesh in order to obtain reliable results in numerical simulations that apply the SST $k-\omega$ model. Comparing both numerical and experimental data, it seems the SST $k$ - $\omega$ model fails to predict with accuracy the potential core at large $H / D$ values. However, a good agreement was obtained for the velocity profile over the target surface and along the jet axis, with a secondary velocity peak identified at $H / D=2$. Regarding the influence of the nozzle-to-plate distance in the Nusselt number, the results show that the maximum value is registered at the stagnation point in all cases, however the value of this peak present small variation between $H / D=2$ to $H / D=7$. It seems that at $\operatorname{Re}<2,000$ the maximum Nusselt number depends only on the Reynolds number. However, a secondary Nusselt number peak is clearly identified at $H / D=2$, showing that the nozzle-to-plate spacing affects the generation of this local maxima. From this study it is concluded that the heat transfer performance increases with the increase of the Reynolds number and the decrease of the $H / D$ value.

\section{Acknowledgements:}

The first author would like to express her gratitude for the support given by the Portuguese Foundation for Science and Technology (FCT) and the MIT Portugal Program. This work has been supported by FCT - Fundação para a Ciência e Tecnologia within the R\&D Units Project Scope: UIDB/00319/2020 (ALGORITMI Center) and R\&D Units Project Scope UIDP/04077/2020 (METRICS Center).

$\begin{array}{ll}\text { Nomenclature } \\ D & \text { nozzle diameter, } \mathrm{m} \\ D_{\omega} & \text { cross-diffusion term } \\ G & \text { generation } \\ h & \text { heat transfer coefficient, } \mathrm{W} /\left(\mathrm{m}^{2} \mathrm{~K}\right)\end{array}$

$H$ nozzle-to-plate distance, $\mathrm{m}$

$k \quad$ turbulence kinetic energy, $\mathrm{m}^{2} / \mathrm{s}^{2}$

$k_{\text {air }}$ Thermal conductivity $\mathrm{W} / \mathrm{mK}$

$\mathrm{Nu}$ Nusselt number

$p$ the pressure, $\mathrm{Pa}$

$\dot{q}$ heat flux, $\mathrm{W} / \mathrm{m}^{2}$

$r$ radial distance, $\mathrm{m}$

Re Reynolds number

$S \quad$ user-defined source term

SST Shear Stress Transport

$t$ time, $\mathrm{s}$

$T$ temperature, ${ }^{\circ} \mathrm{C}$

$U$ time-averaged velocity magnitude, $\mathrm{m} / \mathrm{s}$

$u \quad$ the velocity in $x$-direction, $\mathrm{m} / \mathrm{s}$

$v \quad$ the velocity in $y$-direction, $\mathrm{m} / \mathrm{s}$

$x, y$ cartesian coordinates

$Y$ dissipation

\section{Greek symbols}

$\Gamma \quad$ effective diffusivity

$\alpha \quad$ thermal diffusivity, $\mathrm{m}^{2} / \mathrm{s}$

$\mu$ dynamic viscosity, $\mathrm{m} / \mathrm{s}$

$\omega$ specific dissipation rate, $1 / \mathrm{s}$

\section{Subscripts and superscripts}

jet air jet

wall wall of the target surface

\section{References:}

[1] C. S. Lau, M. Z. Abdullah, and F. Che Ani, "Three dimensional thermal investigations at board level in a reflow oven using thermal-coupling method," Solder. Surf. Mt. Technol., vol. 24, no. 3, pp. 167$182,2012$.

[2] D. C. Whalley, "A simplified model of the reflow soldering process," J. Mater. Process. Technol., vol. 150, pp. 134-144, 2004.

[3] I. Balázs and G. Harsányi, "Heating characteristics of convection reflow ovens," Appl. Therm. Eng., vol. 29, no. 11-12, pp. 2166-2171, 2009.

[4] T. N. Tsai, "Thermal parameters optimization of a reflow soldering profile in printed circuit board assembly: A comparative study," Appl. Soft Comput. J., vol. 12, no. 8, pp. 2601-2613, 2012.

[5] S. Yong, J. Z. Zhang, and G. N. Xie, "Convective heat transfer for multiple rows of impinging air jets with small jet-to-jet spacing in a semi-confined channel," Int. J. Heat Mass Transf., vol. 86, pp. 832-842, 2015.

[6] S. V. Garimella and V. P. Schroeder, "Local Heat Transfer Distributions in Confined Multiple Air Jet Impingement," J. Electron. Packag., vol. 123, no. 3, p. 165, 2001 .

M. Angioletti, R. M. Di Tommaso, E. Nino, and G. 
Ruocco, "Simultaneous visualization of flow field and evaluation of local heat transfer by transitional impinging jets," Int. J. Heat Mass Transf., vol. 46, pp. 1703-1713, 2003.

[8] R. Ben Kalifa, S. Habli, N. M. Saïd, H. Bournot, and G. Le Palec, "Parametric analysis of a round jet impingement on a heated plate," Int. J. Heat Fluid Flow, vol. 57, pp. 11-23, 2016.

[9] S. A. Reodikar, H. C. Meena, R. Vinze, and S. V. Prabhu, "Influence of the orifice shape on the local heat transfer distribution and axis switching by compressible jets impinging on flat surface," Int. J. Therm. Sci., vol. 104, pp. 208-224, 2016.

[10] H. Shariatmadar, S. Mousavian, M. Sadoughi, and M. Ashjaee, "Experimental and numerical study on heat transfer characteristics of various geometrical arrangement of impinging jet arrays," Int. J. Therm. Sci., vol. 102, pp. 26-38, 2016.

[11] R. Brakmann, L. Chen, B. Weigand, and M. Crawford, "Experimental and Numerical Heat Transfer Investigation of an Impinging Jet Array on a Target Plate Roughened by Cubic Micro Pin Fins 1," J. Turbomach., vol. 138, no. 11, p. 111010 , 2016.

[12] J. Lee and S. J. Lee, "The effect of nozzle configuration on stagnation region heat transfer enhancement of axisymmetric jet impingement," Int. J. Heat Mass Transf., vol. 43, no. 18, pp. 34973509, 2000.

[13] R. Viskanta, "Nusselt-Reynolds Prize Paper - Heat Transfer to Impinging Isothermal Gas and Flame Jets," Exp. Therm. Fluid Sci., vol. 6, pp. 111-134, 1993.

[14] Y. Q. Zu, Y. Y. Yan, and J. D. Maltson, “CFD Prediction for Multi-Jet Impingement Heat Transfer," Vol. 3 Heat Transf. Parts A B, pp. 483490, 2009.

[15] J. Ortega-Casanova and F. J. Granados-Ortiz, "Numerical simulation of the heat transfer from a heated plate with surface variations to an impinging jet," Int. J. Heat Mass Transf., vol. 76, pp. 128143, 2014.

[16] Z. Wen, Y. He, X. Cao, and C. Yan, "Numerical study of impinging jets heat transfer with different nozzle geometries and arrangements for a ground fast cooling simulation device," Int. J. Heat Mass Transf., vol. 95, pp. 321-335, 2016.

[17] P. S. Penumadu and A. G. Rao, "Numerical investigations of heat transfer and pressure drop characteristics in multiple jet impingement system," Appl. Therm. Eng., vol. 110, pp. 1511-1524, 2017.

[18] F. R. Menter, "Two-equation eddy-viscosity turbulence models for engineering applications," AIAA J., vol. 32, no. 8, pp. 1598-1605, 1994.

[19] F. R. Menter, J. C. Ferreira, and T. Esch, "The SST Turbulence Model with Improved Wall Treatment for Heat Transfer Predictions in Gas Turbines," Int. Gas Turbine Congr. 2003, no. 1992, pp. 1-7, 2003.

[20] S. Spring, Y. Xing, and B. Weigand, “An
Experimental and Numerical Study of Heat Transfer From Arrays of Impinging Jets With Surface Ribs," J. Heat Transfer, vol. 134, no. 8, p. 082201, 2012.

[21] J. H. Ferziger and M. Peric, Computational Methods for Fluid Dynamics, 3rd ed. USA: Springer, 1996.

[22] Inc. ANSYS, “ANSYS FLUENT Theory Guide," Release 18.2. pp. 1-759, 2013.

[23] Y. A. Cengel and A. J. Ghajar, Heat and Mass Transfer: Fundamentals and Applications. 2011.

[24] P. Xu, A. P. Sasmito, S. Qiu, A. S. Mujumdar, L. $\mathrm{Xu}$, and L. Geng, "Heat transfer and entropy generation in air jet impingement on a model rough surface," Int. Commun. Heat Mass Transf., vol. 72, pp. 48-56, 2016.

[25] S. Caliskan and S. Baskaya, "Experimental investigation of impinging jet array heat transfer from a surface with V-shaped and convergentdivergent ribs," Int. J. Therm. Sci., vol. 59, pp. 234246, 2012.

[26] T. Zhou, D. Xu, J. Chen, C. Cao, and T. Ye, "Numerical analysis of turbulent round jet impingement heat transfer at high temperature difference," Appl. Therm. Eng., vol. 100, pp. 55-61, 2016.

[27] D. W. Zhou and S. J. Lee, "Forced convective heat transfer with impinging rectangular jets," Int. J. Heat Mass Transf., vol. 50, no. 9-10, pp. 19161926, 2007.

[28] M. Angioletti, E. Nino, and G. Ruocco, “CFD turbulent modelling of jet impingement and its validation by particle image velocimetry and mass transfer measurements," Int. J. Therm. Sci., vol. 44, no. 4, pp. 349-356, 2005.

[29] A. Melling, "Tracer particles and seeding for particle image velocimetry," Meas. Sci. Technol., vol. 8, pp. 1406-1416, 1997.

[30] J. Westerweel, "Fundamentals of digital particle image velocimetry," Meas. Sci. Technol., vol. 8, pp. 1379-1392, 1997.

[31] D. Dynamics, "Dynamic Studio, User's Guide," Skovlunde, 2016.

[32] A. Sciacchitano and B. Wieneke, "PIV uncertainty propagation," Meas. Sci. Technol., no. August, p. 84006, 2016.

[33] H. Martin, "Heat and Mass Transfer between Impinging Gas Jets and Solid Surfaces," Adv. Heat Transf., vol. 13, pp. 1-60, 1977.

[34] J. N. B. Livingood and P. Hrycak, "Impingement heat transfer from turbulent air jets to flat plates: A literature survey," Security, vol. X-2778, no. May, p. $43,1973$.

[35] N. Zuckerman and N. Lior, "Jet Impingement Heat Transfer : Physics, Correlations, and Numerical Modeling," Adv. Heat Transf., vol. 39, no. 06, pp. 565-631, 2006. 
[36] H. M. Hofmann, M. Kind, and H. Martin, "Measurements on steady state heat transfer and flow structure and new correlations for heat and mass transfer in submerged impinging jets," Int. J. Heat Mass Transf., vol. 50, no. 19-20, pp. 39573965, 2007.

[37] Y. O. Æ. E. Baydar, "Flow structure and heat transfer characteristics of an unconfined impinging air jet at high jet Reynolds numbers," pp. 13151322, 2008.

[38] L. Xin, L. A. Gabour, and J. H. Lienhard V, "Stagnation-Point Heat Transfer During Impingement of Laminar Liquid Jets : Analysis Including," J. Heat Transfer, vol. 115, no. February, pp. 99-106, 1993.

[39] R. Gardon and C. Akfirat, "Heat Transfer Characteristics of Impinging Two-Dimensional Air Jets," J. Heat Transf. Asme, pp. 1-7, 1966.
[40] V. Katti and S. V Prabhu, "Experimental study and theoretical analysis of local heat transfer distribution between smooth flat surface and impinging air jet from a circular straight pipe nozzle," vol. 51, pp. 4480-4495, 2008.

[41] R. Gardon and C. Akfirat, "The role of turbulence in determining the heat-transfer characteristics of impinging jets," Int. J. Heat Mass Transf., vol. 8, pp. 1261-1272, 1965.

[42] V. Katti and S. V Prabhu, "Experimental study and theoretical analysis of local heat transfer distribution between smooth flat surface and impinging air jet from a circular straight pipe nozzle," Int. J. Heat Mass Transf., vol. 51, pp. 4480-4495, 2008. 